\title{
Evolution of superoscillatory data
}

\author{
Y Aharonov ${ }^{1}$, F Colombo ${ }^{2}$, I Sabadini ${ }^{2}$, D C Struppa ${ }^{1}$ \\ and $\mathrm{J}$ Tollaksen ${ }^{1}$ \\ ${ }^{1}$ Institute of Quantum Studies and Schmid College of Science and Technology, \\ Chapman University, Orange 92866, CA, USA \\ ${ }^{2}$ Politecnico di Milano, Dipartimento di Matematica, Via E. Bonardi, 9 I-20133 \\ Milano, Italy \\ E-mail: fabrizio.colombo@polimi.it
}

Received 9 December 2013, revised 1 April 2014

Accepted for publication 7 April 2014

Published 2 May 2014

\begin{abstract}
Weak measurements and the theory of weak values have a very deep meaning in quantum mechanics, and new phenomena associated with them has recently been observed experimentally. This theory has also directly led to the notion of superoscillating sequences of functions. In this paper we consider Cauchy problems with superoscillatory initial conditions (in particular, the Cauchy problem for the Schrödinger equation and some of its variations), and we give conditions under which the superoscillations persist in time. Our work is based on results from the theory of formal solutions to Cauchy problems and on the study of the specific growth of superoscillatory sequences, when regarded as functions of a complex variable. There are two main aims of this paper: one is to explain the mathematical tools that are necessary to study superoscillations, also repeating a few results that we have already proved in other papers in order to clarify the strategy. The second aim is to show that our technique applies to a large class of problems, showing under which conditions the superoscillatory phenomenon persists. Finally, we point out that our strategy can be applied also to non-constant coefficients differential equations as the quantum harmonic oscillator.
\end{abstract}

Keywords: superoscillations, evolution problem, Schrodinger equation PACS numbers: 42.25.Fx, 06.35.Ge, 42.30.Kq, 02.30.Mv

\section{Introduction}

Quantum physicists have discovered and experimentally demonstrated a very interesting phenomenon which they called superoscillations, see $[1,9,10,13-16]$. In a series of recent 
papers the authors have started the study of superoscillations from the mathematical point of view, see [2-7] and the forthcoming monograph [8].

The aim of this paper is to show how the use of general techniques from the theory of formal solutions to Cauchy problems, combined with ideas from the theory of convolution operators in spaces of holomorphic functions with growth conditions, allows for the study of the evolution of superoscillatory data when taken as initial values for Cauchy problems for a very large class of linear constant coefficients differential equations.

Let us begin by recalling the identity

$$
F_{n}(x, a)=\left(\cos \left(\frac{x}{n}\right)+\mathrm{i} a \sin \left(\frac{x}{n}\right)\right)^{n}=\sum_{k=0}^{n} C_{k}(n, a) \mathrm{e}^{\mathrm{i}(1-2 k / n) x}
$$

for $a \in \mathbb{R}, a>1, x \in \mathbb{R}$ where

$$
C_{k}(n, a)=\left(\begin{array}{l}
n \\
k
\end{array}\right)\left(\frac{1+a}{2}\right)^{n-k}\left(\frac{1-a}{2}\right)^{k},
$$

where $\left(\begin{array}{l}n \\ k\end{array}\right)$ denotes the binomial coefficients. The sequence $\left\{F_{n}(x, a)\right\}$ is said to be superoscillating because while its addends have frequencies $|1-2 k / n| \leqslant 1$, for $n \in \mathbb{N}$, $k=0,1, \ldots, n$, one can show that on every compact set in $\mathbb{R}$, the sequence $\left\{F_{n}(x, a)\right\}$ converges uniformly to $F(x, a)=\mathrm{e}^{\mathrm{i} a x}$, whose frequency $a$ can be arbitrarily large (for the proof see [3]).

A general question we address in this paper is the following. Establish the conditions that we need to put on a differential polynomial $P(D)$, in order for the solution to the Cauchy problem

$$
P(D) \psi(x, t)=0, \quad \psi(x, 0)=\varphi_{n}(x)
$$

to be superoscillating whenever $\varphi_{n}$ is superoscillating.

The main example that guides this discussion is the case in which we consider the Cauchy problem for the Schrödinger equation in the case of a free particle:

$$
\mathrm{i} \frac{\partial \psi(x, t)}{\partial t}=-\frac{\partial^{2} \psi(x, t)}{\partial x^{2}}, \quad \psi(x, 0)=F_{n}(x, a) .
$$

In [5] we have shown that the solution $\psi_{n}(x, t)$, is given by

$$
\psi_{n}(x, t)=\sum_{k=0}^{n} C_{k}(n, a) \mathrm{e}^{\mathrm{i}(1-2 k / n) x} \mathrm{e}^{-\mathrm{i} t(1-2 k / n)^{2}},
$$

and that we have

$$
\lim _{n \rightarrow \infty} \psi_{n}(x, t)=\mathrm{e}^{\mathrm{i} a x-\mathrm{i} a^{2} t}
$$

uniformly on the compact sets of $\mathbb{R}^{2}$. In other words, the solution to the Cauchy problem for the Schrödinger equation with a superoscillating initial datum is still superoscillating.

The crucial point in the previous discussion is the fact that the solution (4) of the Cauchy problem (3) can be written as:

$$
\psi_{n}(x, t)=\sum_{m=0}^{\infty} \frac{(\mathrm{i} t)^{m}}{m !} \frac{\mathrm{d}^{2 m}}{\mathrm{~d} x^{2 m}} F_{n}(x, a)
$$

for every $x \in \mathbb{R}$ and $t \in \mathbb{R}$. To study the superoscillatory phenomenon, when $n$ becomes arbitrarily large, we have to show that the operator

$$
U\left(\frac{\mathrm{d}}{\mathrm{d} x}, t\right):=\sum_{m=0}^{\infty} \frac{(\mathrm{i} t)^{m}}{m !} \frac{\mathrm{d}^{2 m}}{\mathrm{~d} x^{2 m}}
$$


acts continuously on a class of functions that contains $F_{n}(x)$ so that we can write

$$
\lim _{n \rightarrow \infty} U\left(\frac{\mathrm{d}}{\mathrm{d} x}, t\right) F_{n}(x, a)=U\left(\frac{\mathrm{d}}{\mathrm{d} x}, t\right) \lim _{n \rightarrow \infty} F_{n}(x, a)=U\left(\frac{\mathrm{d}}{\mathrm{d} x}, t\right) F(x, a) .
$$

Thus, one can state the problem more in general. Before that, we start by giving the definition of superoscillating sequence.

Definition 1.1. Given the continuous function $f$ represented by the convergent series $f(x)=\sum_{j=0}^{\infty} d_{j} \mathrm{e}^{\mathrm{i} a_{j} x}$, where $d_{j} \in \mathbb{C}$ and $a_{j} \in \mathbb{R}$, we say that the sequence

$$
S_{n}(x)=\sum_{j=0}^{n} C_{j}(n) \mathrm{e}^{\mathrm{i} k_{j}(n) x}
$$

is superoscillating ( $f$-superoscillating when we want to make the limit explicit) if there exists an index $J$ such that $\sup _{j}\left|k_{j}(n)\right|<a_{J}$ and the sequence $S_{n}$ converges uniformly to $f$ on some compact set of $\mathbb{R}$.

Problem 1.2. Identify a significant class of operators $U\left(\frac{\mathrm{d}}{\mathrm{d} x}, t\right)$, including the Schrödinger operator, which acts continuously on a space of functions that contains superoscillating functions.

A strictly related problem arises if we notice that, in the previous computations, the function

$$
Y_{n}(t, a)=\sum_{k=0}^{n} C_{k}(n, a) \mathrm{e}^{-\mathrm{i} t(1-2 k / n)^{2}},
$$

converges uniformly to

$$
Y(t, a)=\mathrm{e}^{-\mathrm{i} a^{2} t},
$$

for all $t \in[-T, T]$, where $T$ is any real positive number. Thus, it is reasonable to ask whether one can solve the following problem.

Problem 1.3. Let $C_{k}(n, a)$ be as in (2), and let $a \in \mathbb{R}, t \in[-T, T]$ where $T$ is any real positive number. Identify a class of analytic functions $G$ such that the sequence

$$
Y_{n}(t, a)=\sum_{k=0}^{n} C_{k}(n, a) \mathrm{e}^{\mathrm{i} t G(-\mathrm{i}(1-2 k / n))}
$$

is superoscillating.

Remark 1.4. Both problems 1.2 and 1.3 can be rigorously solved by studying the Fourier multipliers associated with the symbol of the operator

$$
U\left(\frac{\mathrm{d}}{\mathrm{d} x}, t\right):=\sum_{m=0}^{\infty} a_{m}(t) \frac{\mathrm{d}^{m}}{\mathrm{~d} x^{m}}
$$

that is

$$
\hat{U}(\zeta, t):=\sum_{m=0}^{\infty} a_{m}(t) \zeta^{m}
$$

in suitable spaces of holomorphic functions with growth conditions. 
The philosophy of our approach is the following. We begin by considering a Cauchy problem with superoscillatory initial conditions. We then use standard methods from the theory of formal solutions to Cauchy problems (see section 2) to construct a convergent solution to the problem itself. To do so, we will need to use the specific growth of superoscillating sequences, when regarded as functions of a complex variable. Then, we need to show that the resulting solution maintains a superoscillatory behavior. To this end, we will construct suitable convolution operators on the space of holomorphic functions to which the solutions belong (see section 3), and we will show that it is possible to take the limit of the solutions thus showing that the superoscillatory behavior remains for all times. We will see that the growth conditions that are necessary for this last limiting process are somewhat more restrictive than those that are necessary for the convergence of the formal solutions. Our result is in accordance with [16] in which the authors showed that the persistence time is proportional to $n$, for any fixed, large $n$. In section 4 we use this scheme to study the solutions of some Cauchy problems associated with some differential equations of special type, like a generalized Schrödinger type equation, the heat equation, and a class of equations of non-Kowalevskaya type. Finally, in section 5, we deal with the case of convolution equations.

\section{Formal solutions of Cauchy problems}

The theory of formal solutions of Cauchy problems for linear constant coefficients partial differential equations goes back to the early work of Kowalevskaya who considered, in [21], the characteristic Cauchy problem for the complex heat equation:

$$
\frac{\partial}{\partial t} u(z, t)=\frac{\partial^{2}}{\partial z^{2}} u(z, t), \quad u(z, 0)=\varphi(z),
$$

where $t$ and $z$ are complex variables and $\varphi$ is holomorphic in a neighborhood of the origin. She proved, in particular, that the unique formal solution

$$
u(z, t)=\sum_{m=0}^{\infty} \frac{t^{m}}{m !} \frac{\mathrm{d}^{2 m}}{\mathrm{~d} z^{2 m}} \varphi(z)
$$

converges if and only if $\varphi(z)$ is an entire function of exponential order at most 2, i.e. there are positive constants $A, B$ such that

$$
|\varphi(z)| \leqslant A \exp \left(B|z|^{2}\right) .
$$

Since then, the question of how to construct formal solutions to generalizations of the heat equation, and how to ensure their convergence, has been taken up by many mathematicians, and without pretense of completeness, we will now consider the case, see [11, 19, 23], in which one studies the Cauchy problem associated with the differential equation

$$
\frac{\partial^{r v}}{\partial t^{r \nu}} u(z, t)=\sum_{j=1}^{\nu} a_{j} \frac{\partial^{r(v-j)}}{\partial t^{r(v-j)}} \frac{\partial^{j p}}{\partial z^{j p}} u(z, t)
$$

where $r, p, v \in \mathbb{N}, 1 \leqslant r<p, v \geqslant 1, a_{j} \in \mathbb{C}$ and with initial conditions

$$
\frac{\partial^{\ell}}{\partial t^{\ell}} u(z, 0)=0, \quad \ell=0, \ldots, r v-2, \quad \frac{\partial^{r \nu-1}}{\partial t^{r v-1}} u(z, 0)=\varphi(z) .
$$

Differential equation (5) can be rewritten as (see [19]):

$$
P\left(\frac{\partial}{\partial z}, \frac{\partial}{\partial t}\right)=\prod_{j=1}^{\mu} P_{j}^{\ell_{j}},
$$


where

$$
P_{j}=P_{j}\left(\frac{\partial}{\partial z}, \frac{\partial}{\partial t}\right)=\frac{\partial^{r}}{\partial t^{r}}-\alpha_{j} \frac{\partial^{p}}{\partial z^{p}},
$$

$\ell_{j}, \mu$ are suitable natural numbers, and $\alpha_{j} \in \mathbb{C}$ are the distinct roots of the characteristic equation

$$
\lambda^{\nu}-\sum_{j=1}^{\nu} a_{j} \lambda^{\nu-j}=0
$$

In [19] it is shown that:

Theorem 2.1. The formal solution to the Cauchy problem (5) with initial conditions (6) is given by

$$
\tilde{u}(z, t)=\sum_{m \geqslant r v-1} u_{m}(t z) \frac{t^{m}}{m !}=\sum_{m \geqslant 0} u_{r m+r v-1}(t z) \frac{t^{r m+r v-1}}{(r m+r v-1) !},
$$

where

$$
u_{r m+r v-1}(z)=A(m) \frac{\mathrm{d}^{p m}}{\mathrm{~d} z^{p m}} \varphi(z), \quad m \geqslant 0
$$

and $A(m)$ can be computed by solving a suitable difference equation.

Remark 2.2. It turns out that the coefficients $A(m)$ are of the form

$$
A(m)=\sum_{j=1}^{\mu} \alpha_{j}^{m} \sum_{k=1}^{\ell_{j}} c_{j k} m^{k-1}, \quad m \geqslant 0,
$$

where $\ell_{j}, \mu, \alpha_{j}$ are as above, see again [19] for the details.

Remark 2.3. Theorem 2.1 and remark 2.2 yield that the formal solution to the Cauchy problem (5) with initial conditions (6) is given by

$$
\tilde{u}(z, t)=\sum_{m \geqslant 0} t^{r m+r \nu-1} \frac{A(m)}{(r m+r \nu-1) !} \frac{\mathrm{d}^{p m}}{\mathrm{~d} z^{p m}} \varphi(z):=U\left(\frac{\mathrm{d}}{\mathrm{d} z}, t\right) \varphi(z) .
$$

Remark 2.4. We would like to point out that the use of complex numbers is not simply a matter of elegance, but it is indeed necessary to completely justify the formal calculations that are used. In particular, in the sections to follow, we will show how to make our formal calculations rigorous.

To motivate the subsequent analysis we will now consider in detail the Cauchy problem for the Schrödinger equation

$$
\mathrm{i} \frac{\partial \psi(x, t)}{\partial t}=-\frac{\partial^{2} \psi(x, t)}{\partial x^{2}}, \quad \psi(x, 0)=F_{n}(x, a) .
$$

The following result is easily established by using the Fourier transform.

Theorem 2.5. The time evolution of the spatial superoscillating function $F_{n}(x, a)$, is given by

$$
\psi_{n}(x, t)=\sum_{k=0}^{n} C_{k}(n, a) \mathrm{e}^{\mathrm{i} x(1-2 k / n)} \mathrm{e}^{-\mathrm{i} t(1-2 k / n)^{2}} .
$$

The crucial fact in our approach is that $\psi_{n}(x, t)$ can be written as described below (see theorem 3.1 in [5]): 
Theorem 2.6. The function

$$
\psi_{n}(x, t)=\sum_{k=0}^{n} C_{k}(n, a) \mathrm{e}^{\mathrm{i} x(1-2 k / n)} \mathrm{e}^{-\mathrm{i} t(1-2 k / n)^{2}}
$$

can be written as

$$
\psi_{n}(x, t)=\sum_{m=0}^{\infty} \frac{(\mathrm{i} t)^{m}}{m !} \frac{\mathrm{d}^{2 m}}{\mathrm{~d} x^{2 m}} F_{n}(x, a)
$$

for every $x \in \mathbb{R}$ and $t \in \mathbb{R}$.

The idea of the proof is to consider the expansion

$$
\mathrm{e}^{-\mathrm{i} t(1-2 k / n)^{2}}=\sum_{m=0}^{\infty} \frac{\left[-\mathrm{i} t(1-2 k / n)^{2}\right]^{m}}{m !}
$$

and to observe that it can be written as

$$
\begin{aligned}
\psi_{n}(x, t) & =\sum_{m=0}^{\infty} \frac{(\mathrm{i} t)^{m}}{m !} \sum_{k=0}^{n} C_{k}(n, a) \frac{\mathrm{d}^{2 m}}{\mathrm{~d} x^{2 m}} \mathrm{e}^{\mathrm{i} x(1-2 k / n)} \\
& =\sum_{m=0}^{\infty} \frac{(\mathrm{i} t)^{m}}{m !} \frac{\mathrm{d}^{2 m}}{\mathrm{~d} x^{2 m}} \sum_{k=0}^{n} C_{k}(n, a) \mathrm{e}^{\mathrm{i} x(1-2 k / n)} \\
& =\sum_{m=0}^{\infty} \frac{(\mathrm{i} t)^{m}}{m !} \frac{\mathrm{d}^{2 m}}{\mathrm{~d} x^{2 m}} F_{n}(x, a) .
\end{aligned}
$$

This result is consistent with the discussion we had regarding the formal solutions to the Cauchy problem.

The main problem is now to take the limit for $n \rightarrow+\infty$ of $\psi_{n}(x, t)$. Let us take, formally, the limit and recall that

$$
F_{n}(x, a) \rightarrow \mathrm{e}^{\mathrm{i} a x}
$$

so we obtain, see [5],

$$
\psi(x, t)=\sum_{m=0}^{\infty} \frac{(\mathrm{i} t)^{m}}{m !} \frac{\mathrm{d}^{2 m}}{\mathrm{~d} x^{2 m}} \mathrm{e}^{\mathrm{i} a x}=\mathrm{e}^{\mathrm{i} a x-i a^{2} t} .
$$

In order to fully justify these formal computations, see theorem 3.9, we will now study in some detail the properties of multipliers on spaces of holomorphic functions with growth conditions.

\section{Spaces of holomorphic functions with growth}

In this section we collect some well known definitions and results for infinite-order differential operators, as well as for convolution operators on spaces of holomorphic functions satisfying suitable growth conditions.

Infinite-order differential operators are a very special case of convolution equations, and we begin our discussion with them as a motivation.

Definition 3.1. An entire function $F: \mathbb{C} \rightarrow \mathbb{C}$ is said to be of infraexponential type if for any $\varepsilon>0$ there exists $A_{\varepsilon}>0$ such that

$$
|F(z)| \leqslant A_{\varepsilon} \exp (\varepsilon|z|) \text {. }
$$

We denote by $\operatorname{Exp}_{0}(\mathbb{C})$ the space of entire functions of infraexponential type. 
Remark 3.2. As it is well known, see [12, 20], an entire function of the form

$$
g(\zeta):=\sum_{m=0}^{\infty} b_{m} \zeta^{m}
$$

is of infraexponential type if and only if its coefficients satisfy the condition

$$
\lim _{k \rightarrow \infty} \sqrt[k]{\left|b_{k}\right| k !}=0
$$

Functions of infraexponentialy type are very helpful in constructing the class of differential operators of infinite order. Specifically, see [20], an operator of the form

$$
g\left(\frac{\mathrm{d}}{\mathrm{d} z}\right):=\sum_{m=0}^{\infty} b_{m} \frac{\mathrm{d}^{m}}{\mathrm{~d} z^{m}}
$$

is an infinite-order differential operator, also called local operator, which acts continuously on holomorphic functions in $\mathbb{C}$ if and only if

$$
\lim _{k \rightarrow \infty} \sqrt[k]{\left|b_{k}\right| k !}=0
$$

It is natural to ask whether entire functions with growth faster than infraexponential can still give rise to local operators. The answer is negative, but it can be shown that it is possible to use such functions to define more general operators, called convolution operators. The simplest such example is given by

$$
g(\zeta)=\sum_{m=0}^{\infty} \frac{1}{m !} \zeta^{m}=\mathrm{e}^{\zeta}
$$

which is the symbol of the unit translation operator and which is nothing but the convolution with the Dirac delta centered at $z=-1$.

In order to consider the general case, where functions of different growth are considered (such as they appear in section 2), one needs some more refined techniques, which we briefly sketch here, while referring the mathematically inclined reader to [18, 24] for a detailed description.

The key ingredient is the notion of analytically uniform space (AU-space), for which we give a simplified description. A topological vector space of (generalized) functions $X$ is said to be an AU-space if its dual $X^{\prime}$ is isomorphic (via some version of the Fourier transform) to a space of entire functions with suitable growth at infinity. The main example that one should keep in mind is the case of $X=\mathcal{H}\left(\mathbb{C}^{n}\right)$ the space of entire functions on $\mathbb{C}^{n}$. In this case $X^{\prime}$ is the space of analytic functionals, and its dual is the space

$$
\operatorname{Exp}\left(\mathbb{C}^{n}\right)=\left\{f \in \mathcal{H}\left(\mathbb{C}^{n}\right): \exists A, B>0:|f(z)| \leqslant A \exp (B|z|)\right\}
$$

of entire functions of exponential type: in this case the transform that realizes the isomorphism between the space of analytic functionals and the space of entire functions of exponential type is the Fourier-Borel transform. The general theory of AU-spaces now says that if we consider a continuous multiplicator on $\operatorname{Exp}\left(\mathbb{C}^{n}\right)$, i.e. a function $F$ such that the product by $F$ acts continuously on $\operatorname{Exp}\left(\mathbb{C}^{n}\right)$, then $F$ can be interpreted as the symbol for a convolution operator on $\mathcal{H}\left(\mathbb{C}^{n}\right)$. Thus, for example, the function $\mathrm{e}^{\zeta}$ which we have considered above, acts as a multiplier on $\operatorname{Exp}\left(\mathbb{C}^{n}\right)$, and therefore it is the symbol of a convolutor on the space of entire functions, as we saw earlier.

It is interesting to see what happens if we consider smaller subspaces of $\mathcal{H}$, and we look at the resulting space of convolutors, which we expect to be a larger space than functions of exponential type. The spaces we are interested in are of two types: we will describe them both, 
and will state the duality theorem that fully characterizes the symbols of the convolutors on them. The ideas we are going to use were originally put forward in [24].

For any positive real number $\rho$, we define two spaces, each of which has growth of finite order, but different type (see e.g. [22]).

Definition 3.3. The space

$$
A_{\rho}:=\left\{f \in \mathcal{H}(\mathbb{C}): \exists A, B>0:|f(z)| \leqslant A \exp \left(B|z|^{\rho}\right)\right\},
$$

is said to be the space of functions of order $\rho$ and finite type.

Correspondingly we have

Definition 3.4. The space

$$
A_{\rho, 0}:=\left\{f \in \mathcal{H}(\mathbb{C}): \forall \varepsilon>0, \exists A_{\varepsilon}>0:|f(z)| \leqslant A_{\varepsilon} \exp \left(\varepsilon|z|^{\rho}\right)\right\},
$$

is said to be the space of functions of order $\rho$ and minimal type.

Note that with this notation, $\operatorname{Exp}(\mathbb{C})=A_{1}$ and $\operatorname{Exp}_{0}(\mathbb{C})=A_{1,0}$.

The following theorem, see [24], makes the results in the previous section more precise.

Theorem 3.5. Let $\rho$ be a real number such that, $\rho>1$. Then there is a topological isomorphism between the space $A_{\rho}$ and the dual of the space $A_{\rho^{\prime}, 0}$, where

$$
\frac{1}{\rho}+\frac{1}{\rho^{\prime}}=1
$$

Conversely, there is a topological isomorphism between the space $A_{\rho, 0}$ and the dual of the space $A_{\rho}$.

In other words, the elements of $A_{\rho, 0}$ can be viewed as symbols for convolutors acting continuously on $A_{\rho^{\prime}}$ and vice versa the elements of $A_{\rho^{\prime}, 0}$ can be viewed as symbols for convolutors acting continuously on $A_{\rho}$.

Remark 3.6. To illustrate how theorem 3.5 applies to our discussion, let us consider a generalized Schrödinger equation, such as (11) in the sequel, in other words, let us consider an operator of the form

$$
\sum_{m=0}^{\infty} \frac{(\mathrm{i} t)^{m}}{m !} \frac{\mathrm{d}^{m p}}{\mathrm{~d} x^{m p}}
$$

The symbol of this operator belongs to $A_{p}$, thus by theorem 3.5 this operator acts continuously on a class of functions with suitable growth conditions, namely $A_{p^{\prime}, 0}$ with $1 / p+1 / p^{\prime}=1$, so that it is possible to commute the series with the limit for $n \rightarrow \infty$ when the initial datum for a Cauchy problem belongs to $A_{p^{\prime}, 0}$.

The theory of AU-spaces is, however, more general than what we have described so far, and we need to offer two more examples of the duality which we have described, and that will play a fundamental role when we generalize our study to the case in which we are solving the Cauchy problem for convolution equations of the special type

$$
\frac{\partial \psi(x, t)}{\partial t}=\mu_{x} * \psi(x, t),
$$

where $\mu_{x}$ is a suitable convolutor in the variable $x$. Without unnecessary generality, we state here the two relevant propositions, which are described in detail in [24]. 
Proposition 3.7. Let $\Delta_{R}=\{\zeta \in \mathbb{C}|| \zeta \mid<R\}$. If

$$
X=\operatorname{Exp}_{1}(\mathbb{C}):=\left\{f \in \mathcal{H}(\mathbb{C}):|f(z)| \leqslant A|z|^{n} \mathrm{e}^{|z|} \text { for some } n\right\}
$$

then the Fourier-Borel transform establishes a topological isomorphism between $X^{\prime}$ and

$$
\mathcal{F} X^{\prime}=\left\{f \in \mathcal{H}\left(\Delta_{1}\right) \text { and } f^{(n)} \text { are uniformly continuous for all } n \in \mathbb{N}\right\} .
$$

Proposition 3.8. Let $R>0$ and let $X$ be the space of entire functions of exponential type less that $R$, i.e.

$$
X=\left\{f \in \mathcal{H}(\mathbb{C}): \exists \varepsilon>0:|f(z)| \leqslant A \mathrm{e}^{(R-\varepsilon)|z|} \text { for some } A>0\right\} .
$$

Then the space of its Fourier-Borel transform $\mathcal{F} X^{\prime}$ is isomorphic to the space of functions holomorphic in the disc $\Delta_{R}$.

We close this section by showing how these ideas can be used to justify the formal calculations for the case of the Schrödinger equation that we carried out in section 2.

Theorem 3.9. Consider the Cauchy problem

Its solution

$$
\mathrm{i} \frac{\partial \psi(x, t)}{\partial t}=-\frac{\partial^{2} \psi(x, t)}{\partial x^{2}}, \quad \psi(x, 0)=F_{n}(x, a) .
$$

$$
\psi_{n}(x, t)=\sum_{k=0}^{n} C_{k}(n, a) \mathrm{e}^{\mathrm{i} x(1-2 k / n)} \mathrm{e}^{-\mathrm{i} t(1-2 k / n)^{2}}
$$

converges, for $n \rightarrow \infty$, and uniformly on compact sets, to $\psi(x, t)=\mathrm{e}^{\mathrm{i} a x-\mathrm{i} a^{2} t}$.

Proof. Indeed, we have seen that the solution

$$
\psi_{n}(x, t)=\sum_{m=0}^{\infty} \frac{(\mathrm{i} t)^{m}}{m !} \frac{\mathrm{d}^{2 m}}{\mathrm{~d} x^{2 m}} F_{n}(x, a)
$$

is obtained by applying the convolutor $\sum_{m=0}^{\infty} \frac{(\mathrm{i} t)^{m}}{m !} \frac{\mathrm{d}^{2 m}}{\mathrm{~d} x^{2 m}}$ to the superoscillating function $F_{n}(x, a)$. Since the convolutor has symbol $\mathrm{e}^{t \zeta^{2}}$, which is an element of $A_{2}$, we see that the limit can be taken inside the series as long as the initial condition is a function in $A_{2,0}$. But the functions $F_{n}$ are finite sums of linear exponentials, and therefore they certainly belong to $A_{2,0}$, which concludes the proof.

\section{Some classes of differential equations}

In this section we solve the Cauchy problem with a given superoscillatory datum for a generalized Schrödinger equation, for the heat equation and, finally, and for some differential equations of non-Kowalevskaya type which are special cases of equation (5), also discussing the persistence of the superoscillatory behavior of the solutions.

\subsection{Generalized Schrödinger equation}

The previous discussion on the Schrödinger equation can be extended to the more general situation in which we replace the second order derivative in Schrödinger equation with the 
derivative of order $p$. This case is important because it shows that a superoscillatory initial datum persists in time for such a class of differential equations.

In what follows it is necessary to distinguish between the case $p$ even and $p$ odd.

\section{Theorem 4.1.}

(a) Consider, for $p$ even, the Cauchy problem for the modified Schrödinger equation

$$
\mathrm{i} \frac{\partial \psi(x, t)}{\partial t}=-\frac{\partial^{p} \psi(x, t)}{\partial x^{p}}, \quad \psi(x, 0)=F_{n}(x, a) .
$$

Then the solution $\psi_{n}(x, t ; p)$, is given by

$$
\psi_{n}(x, t ; p)=\sum_{k=0}^{n} C_{k}(n, a) \mathrm{e}^{\mathrm{i} x(1-2 k / n)} \mathrm{e}^{\mathrm{i} t(-\mathrm{i}(1-2 k / n))^{p}} .
$$

For all $t \in[-T, T]$, where $T$ is any real positive number, we have

$$
\lim _{n \rightarrow \infty} \psi_{n}(x, t ; p)=\mathrm{e}^{\mathrm{i} t(-\mathrm{i} a)^{p}} \mathrm{e}^{\mathrm{i} a x},
$$

for $x \in K$, where $K$ is any compact set in $\mathbb{R}$.

(b) Consider, for $p$ odd, the Cauchy problem for the modified Schrödinger equation

$$
\frac{\partial \psi(x, t)}{\partial t}=\frac{\partial^{p} \psi(x, t)}{\partial x^{p}}, \quad \psi(x, 0)=F_{n}(x, a) .
$$

Then the solution $\psi_{n}(x, t ; p)$, is given by

$$
\psi_{n}(x, t ; p)=\sum_{k=0}^{n} C_{k}(n, a) \mathrm{e}^{\mathrm{i}(1-2 k / n) x} \mathrm{e}^{t(-\mathrm{i}(1-2 k / n))^{p}} .
$$

Moreover, for all $t \in[-T, T]$, where $T$ is any real positive number, we have

$$
\lim _{n \rightarrow \infty} \psi_{n}(x, t, p)=\mathrm{e}^{t(-\mathrm{i} a)^{p}} \mathrm{e}^{\mathrm{i} a x},
$$

for $x \in K$, where $K$ is any compact set in $\mathbb{R}$.

Proof. We give a sketch of the proof of point (a) and we refer the reader to [6] for the proof of (b) and for all the details. Let us consider the Fourier and anti-Fourier transforms of two functions $\psi$ and $g$ respectively:

$$
\mathcal{F}[\psi(x, t)]:=\int_{\mathbb{R}} \psi(x, t) \mathrm{e}^{-\mathrm{i} \lambda x} \mathrm{~d} x, \quad \mathcal{F}^{-1}[g(\lambda, t)]:=\frac{1}{2 \pi} \int_{\mathbb{R}} g(\lambda, t) \mathrm{e}^{\mathrm{i} \lambda x} \mathrm{~d} \lambda .
$$

We set, for simplicity, $\mathcal{F}[\psi(x, t)]=\hat{\psi}(\lambda, t)$. We work in the space of the tempered distributions $\mathcal{S}^{\prime}(\mathbb{R})$ and use a standard Fourier transform argument to solve the Cauchy problem (11). We have

$$
\mathrm{i} \frac{\mathrm{d} \hat{\psi}(\lambda, t)}{\mathrm{d} t}=-(-\mathrm{i} \lambda)^{p} \hat{\psi}(\lambda, t)
$$

and integrating we obtain

$$
\hat{\psi}(\lambda, t)=C(\lambda) \mathrm{e}^{\mathrm{i}(-\mathrm{i} \lambda)^{p} t}
$$

where the arbitrary function $C(\lambda)$ is determined by the initial condition

$$
C(\lambda)=2 \pi \sum_{k=0}^{n} C_{k}(n, a) \delta(\lambda-(1-2 k / n)) .
$$


Thus we have

$$
\hat{\psi}_{n}(\lambda, t ; p)=2 \pi \sum_{k=0}^{n} C_{k}(n, a) \delta(\lambda-(1-2 k / n)) \mathrm{e}^{\mathrm{i}(-\mathrm{i} \lambda)^{p} t} .
$$

Taking now the inverse Fourier transform we obtain $\psi_{n}(x, t)$.

To compute the limit $\lim _{n \rightarrow \infty} \psi_{n}(x, t)$ we write:

$$
\mathrm{e}^{\mathrm{i} t(-\mathrm{i}(1-2 k / n))^{p}}=\sum_{m=0}^{\infty} \frac{\left[\mathrm{i} t(-\mathrm{i}(1-2 k / n))^{p}\right]^{m}}{m !},
$$

and, with some computations, we have

$$
\psi_{n}(x, t ; p)=\sum_{m=0}^{\infty} \frac{(\mathrm{i} t)^{m}}{m !} \sum_{k=0}^{n} C_{k}(n, a) \frac{\mathrm{d}^{m p}}{\mathrm{~d} x^{m p}} \mathrm{e}^{\mathrm{i} x(1-2 k / n)}=\sum_{m=0}^{\infty} \frac{(\mathrm{i} t)^{m}}{m !} \frac{\mathrm{d}^{m p}}{\mathrm{~d} x^{m p}} F_{n}(x, a) .
$$

Passing to the limit as we did for the Schrödinger equation but replacing the spaces $A_{2}$ and $A_{2,0}$ by $A_{p}$ and $A_{p^{\prime}, 0}$ respectively, with $\frac{1}{p}+\frac{1}{p^{\prime}}=1$, we obtain

$$
\psi(x, t ; p)=\lim _{n \rightarrow \infty} \psi_{n}(x, t ; p)=\sum_{m=0}^{\infty} \frac{(\mathrm{i} t)^{m}}{m !} \frac{\mathrm{d}^{m p}}{\mathrm{~d} x^{m p}} \mathrm{e}^{\mathrm{i} a x} .
$$

Since

$$
\psi(x, t)=\sum_{m=0}^{\infty} \frac{(\mathrm{i} t)^{m}}{m !}(\mathrm{i} a)^{m p} \mathrm{e}^{\mathrm{i} a x}
$$

we finally have

$$
\psi(x, t ; p)=\sum_{m=0}^{\infty} \frac{\left((\mathrm{i} a)^{p} \mathrm{i} t\right)^{m}}{m !} \mathrm{e}^{\mathrm{i} a x}=\mathrm{e}^{\mathrm{i} t(\mathrm{i} a)^{p}} \mathrm{e}^{\mathrm{i} a x} .
$$

The following corollary of theorem 4.1 shows how to construct other classes of superoscillating functions:

Corollary 4.2. Let $a>1$, p even, and let $T$ be a real positive number. Then, for all $t \in[-T, T]$, the sequence

$$
\varphi_{n}(t)=\sum_{k=0}^{n} C_{k}(n, a) \mathrm{e}^{\mathrm{i} t(-\mathrm{i}(1-2 k / n))^{p}}
$$

is $\mathrm{e}^{\mathrm{i} t(-\mathrm{i} a)^{p}}$-superoscillating, i.e. we have

$$
\lim _{n \rightarrow \infty} \varphi_{n}(t)=\mathrm{e}^{\mathrm{i} t(-\mathrm{i} a)^{p}} .
$$

\subsection{Heat equation}

We now consider, following the lines of the previous discussion, a Cauchy problem for the heat equation:

$$
\frac{\partial}{\partial t} \psi(x, t)=\frac{\partial^{2}}{\partial z^{2}} \psi(x, t), \quad \psi(x, 0)=F_{n}(x, a) .
$$

With techniques similar to those used to treat the Schrödinger equation, we deduce that the formal solution to this problem is, see (8):

$$
\psi_{n}(x, t)=\sum_{m \geqslant 0} \frac{t^{m}}{m !} \frac{\mathrm{d}^{2 m}}{\mathrm{~d} x^{2 m}} F_{n}(x, a) .
$$


Computations similar to those done in the proof of theorem 4.1, and noticing again that the symbol of the operator belongs to $A_{2}$, show that for all $t \in[-T, T], T>0$

$$
\lim _{n \rightarrow \infty} \psi_{n}(x, t)=\mathrm{e}^{-a^{2} t} \mathrm{e}^{\mathrm{i} a x},
$$

thus the superoscillation is damped in time.

\subsection{Differential equations of non-Kowalevskaya type}

Let us now go back to the differential equation (5) whose formal solution is given in theorem 2.1, and let us study some special cases in which we can guarantee that, when we assign a superoscillatory initial condition in the Cauchy problem (6) we have that the solution to (5) is superoscillating. We consider the case $v=r=1$.

Theorem 4.3. The solution $\psi_{n}(z, t)$ to the Cauchy problem

is such that

$$
\left\{\begin{array}{l}
\frac{\partial}{\partial t} \psi(x, t)=a_{1} \frac{\partial^{p}}{\partial z^{p}} \psi(x, t) \\
\psi(x, 0)=F_{n}(x, a)
\end{array}\right.
$$

$$
\psi(x, t)=\lim _{n \rightarrow+\infty} \psi_{n}(x, t)=\mathrm{e}^{t a_{1}(\mathrm{i} a)^{p}} \mathrm{e}^{\mathrm{i} a x}
$$

and $\psi(z, t)$ is superoscillating in time for:

(i) $p$ even, and $a_{1}$ purely imaginary or $a_{1}=\alpha+\mathrm{i} \beta$ and (ia) $)^{p}>0$, in which case the superoscillation is amplified, or $a_{1}=\alpha+\mathrm{i} \beta$ and $(\mathrm{i} a)^{p} \alpha<0$, in which case the superoscillation is damped.

(ii) $p$ odd, and $a_{1}$ real or $a_{1}=\alpha+\mathrm{i} \beta$ and $\mathrm{i}(\mathrm{i} a)^{p} \beta>0$, in which case the superoscillation is amplified, or $a_{1}=\alpha+\mathrm{i} \beta$ and $\mathrm{i}(\mathrm{i} a)^{p} \beta<0$, in which case the superoscillation is damped.

Proof. To prove the result we regard the previous problem in the complex case and thus we substitute $x$ with a complex variable $z$. Then we know from theorem 2.1 that the formal solution is

$$
\psi_{n}(z, t)=\sum_{m \geqslant 0} t^{m} \frac{A(m)}{m !} \frac{\mathrm{d}^{p m}}{\mathrm{~d} z^{p m}} F_{n}(z, a)
$$

where $A(m)=a_{1}^{m}$ since the characteristic equation is $\lambda-a_{1}=0$. By taking the limit, see theorem 3.5 , we have

$$
\begin{aligned}
\lim _{n \rightarrow+\infty} \psi_{n}(z, t) & =\sum_{m \geqslant 0} t^{m} \frac{a_{1}^{m}}{m !} \frac{\mathrm{d}^{p m}}{\mathrm{~d} z^{p m}} F_{n}(z, a) \\
& =\sum_{m \geqslant 0} t^{m} \frac{a_{1}^{m}}{m !} \frac{\mathrm{d}^{p m}}{\mathrm{~d} z^{p m}} \mathrm{e}^{\mathrm{i} a z} \\
& =\sum_{m \geqslant 0}\left(a_{1} t\right)^{m} m !(\mathrm{i} a)^{p m} \mathrm{e}^{\mathrm{i} a z} \\
& =\mathrm{e}^{t a_{1}(\mathrm{i} a)^{p}} \mathrm{e}^{\mathrm{i} a z} .
\end{aligned}
$$

Assume first that $p$ is even. Then $\mathrm{e}^{t a_{1}(\mathrm{i} a)^{p}}=\mathrm{e}^{(-1)^{p / 2} t a_{1} a^{p}}$, thus if $a_{1}$ is purely imaginary the superoscillation persists in time. If $a_{1}=\alpha+\mathrm{i} \beta$ then

$$
\mathrm{e}^{t a_{1}(\mathrm{i} a)^{p}}=\mathrm{e}^{t(\alpha+\mathrm{i} \beta)(\mathrm{i} a)^{p}}=\mathrm{e}^{t \alpha(\mathrm{i} a)^{p}} \mathrm{e}^{\mathrm{i} t \beta(\mathrm{i} a)^{p}} .
$$


The factor $\mathrm{e}^{t \alpha(\mathrm{i} a)^{p}}$ is amplifying or damping the superoscillation according to the sign of $\alpha(\mathrm{i} a)^{p}$.

If $p$ is odd then $\mathrm{e}^{t_{1}(\mathrm{i} a)^{p}}=\mathrm{e}^{\mathrm{i}(-1)^{(p-1) / 2 t a_{1} a^{p}}}$, thus if $a_{1}$ is real the superoscillation persists in time. If $a_{1}=\alpha+\mathrm{i} \beta$ then

$$
\mathrm{e}^{t a_{1}(\mathrm{i} i)^{p}}=\mathrm{e}^{t(\alpha+\mathrm{i} \beta)(\mathrm{i} a)^{p}}=\mathrm{e}^{t \alpha(\mathrm{i} a)^{p}} \mathrm{e}^{\mathrm{i} t \beta(\mathrm{i} a)^{p}} .
$$

The factor $\mathrm{e}^{\mathrm{i} \beta(\mathrm{i} i)^{p}}$ is amplifying or damping the superoscillation according to the sign of $\mathrm{i} \beta(\mathrm{i} a)^{p}$.

In order to extend this result to a more general differential equation we consider the symbol $g(\zeta, t)$ of the operator $U\left(\frac{\mathrm{d}}{\mathrm{d} z}, t\right)$ :

$$
g(\zeta, t)=\sum_{m \geqslant 0} t^{r m+r \nu-1} \frac{A(m)}{(r m+r v-1) !} \zeta^{p m}
$$

We have the following result:

Proposition 4.4. The order of the function

$$
g(\zeta, t)=\sum_{m \geqslant 0} t^{r m+r v-1} \frac{A(m)}{(r m+r v-1) !} \zeta^{p m}
$$

as holomorphic function in $\zeta$ is $p / r$. As a consequence, $g(t, \zeta)$ turns out to be entire in $\zeta$.

Proof. The order $\rho$ of the function $g$ can be computed using formula (1.05) in [22]: for an entire functions of the form $f(z)=\sum_{m \geqslant 0} c_{m} z^{m}$ the order is

$$
\rho=\frac{m \log m}{\log \left|\frac{1}{c_{m}}\right|} .
$$

In the case of the function $g(\zeta)$ the formula gives, for $t \in[-T, T]$ :

$$
\rho=\varlimsup_{m \rightarrow \infty} \frac{p m \log (p m)}{\log \left(\frac{(r m+r v-1) !}{\left|A(m) t^{r m+r v-1}\right|}\right)} .
$$

By applying the Stirling formula to the factorial, we can rewrite as follows:

$$
\begin{aligned}
\rho & =\varlimsup_{m \rightarrow \infty} \frac{p m(\log (m)+\log (p))}{(r m+r v-1) \log (r m+r v-1)-\log \left(|A(m)||t|^{r m+r v-1}\right)} \\
& =\varlimsup_{m \rightarrow \infty} \frac{p m(\log (m)+\log (p))}{r m(\log (m)+\log (r))-\log \left(|\alpha|^{m} m^{M}\right)-(r m+r v-1) \log (|t|)} \\
& =\varlimsup_{m \rightarrow \infty} \frac{p m \log (m)}{r m \log (m)-m \log (|\alpha|)-M \log (m)-r m \log (|t|)}=\frac{p}{r} .
\end{aligned}
$$

The next result shows that our technique works also for differential equations which contain also higher order derivatives with respect to time.

Theorem 4.5. The Cauchy problem associated with the differential equation

$$
\frac{\partial^{r \nu}}{\partial t^{r \nu}} \psi(z, t)=\sum_{j=1}^{\nu} a_{j} \frac{\partial^{r(v-j)}}{\partial t^{r(v-j)}} \frac{\partial^{j p}}{\partial z^{j p}} \psi(z, t)
$$

where $r, p, v \in \mathbb{N}, 1 \leqslant r<p, v \geqslant 1, a_{j} \in \mathbb{C}$ and with initial conditions

$$
\frac{\partial^{\ell}}{\partial t^{\ell}} \psi(z, 0)=0, \quad \ell=0, \ldots, r v-2, \quad \frac{\partial^{r \nu-1}}{\partial t^{r \nu-1}} \psi(z, 0)=F_{n}(z, a)
$$


has formal solution

$$
\psi_{n}(z, t)=\sum_{m \geqslant r v-1} u_{m, n}(t z) \frac{t^{m}}{m !}=\sum_{m \geqslant 0} u_{r m+r v-1, n}(t z) \frac{t^{r m+r \nu-1}}{(r m+r v-1) !},
$$

where

$$
u_{r m+r v-1, n}(z)=A(m) F_{n}^{(p m)}(z, a), \quad m \geqslant 0 .
$$

Moreover, this formal solution actually converges to a function $\psi$ and it is possible to compute its limit when $n \rightarrow \infty$ to be the function

$$
\psi(z, t)=\sum_{m \geqslant r v-1} u_{m}(t z) \frac{t^{m}}{m !}=\sum_{m \geqslant 0} u_{r m+r v-1}(t z) \frac{t^{r m+r \nu-1}}{(r m+r v-1) !},
$$

where

$$
u_{r m+r \nu-1}(z)=A(m) F^{(p m)}(z, a), \quad m \geqslant 0 .
$$

Proof. Indeed, we have seen that the solution $\psi_{n}(x, t)$ given in (16) is obtained by applying the convolutor

$$
\sum_{m=0}^{\infty} \frac{t^{r m+r v-1}}{(r m+r v-1) !} A(m) \frac{\mathrm{d}^{p m}}{\mathrm{~d} x^{p m}}
$$

to the superoscillating function $F_{n}(x, a)$. Since the symbol of the convolutor has order $p$, see proposition 4.4 , and thus it belongs to $A_{p}$, we see that the limit can be taken inside the series as long as the initial condition is a function in $A_{p^{\prime}, 0}$ and the statement follows.

\section{The case of convolution equations}

We conclude this paper by considering a special kind of convolution equations in which the convolutor acts only on the space variable, while the derivative with respect to $t$ is of the first order. This approach will allow us to answer in the largest possible sense problem 1.2, and will give a very large class of superoscillating sequences.

Specifically, we will consider convolution equations of the form

$$
\frac{\partial \psi(x, t)}{\partial t}=\mu_{x} * \psi(x, t)
$$

for some suitable convolutor $\mu_{x}$ that only acts on the variable $x$. There is a subtle point here, in the sense that if we consider the function $\psi$ to be an infinitely differentiable function in $x$, we can only consider compactly supported distribution as convolutors $\mu_{x}$. However, we know that the functions on which we want to act are actually analytic on $\mathbb{R}$ and, what is more, they extend to entire functions on all of $\mathbb{C}$, because we know they are simply finite sums of exponentials. For this reason, we can consider instead the convolution equation defined on entire functions by

$$
\frac{\partial \psi(z, t)}{\partial t}=\mu_{z} * \psi(z, t)
$$

and now we can interpret the symbol of $\mu_{z}$ as an entire function of suitable growth. Thus, let us consider a convolution equation as follows. Let $\left\{a_{p}\right\}$ be a sequence of complex numbers and consider the convolution equation formally defined by 


$$
\mathrm{i} \frac{\partial \psi(z, t)}{\partial t}=-\sum_{p=0}^{\infty} a_{p} \frac{\partial^{p} \psi(z, t)}{\partial z^{p}} .
$$

The operator on the right-hand side is clearly the infinite sum of the operators for which we have already shown that the superoscillatory behavior of initial data persists. We then define the operator:

$$
U_{p}(t)=\sum_{m=0}^{\infty} \frac{(\mathrm{i} t)^{m}}{m !} \frac{\mathrm{d}^{p m} f}{\mathrm{~d} z^{p m}},
$$

where according to $p \in \mathbb{N}$ one might have to replace it by $-\mathrm{i} t$ as we did in the case of the generalized Schrödinger equation. Now we need to study the operator that formally can be written as the infinite product of the operators $U_{p}$, namely the new operator

$$
U_{\infty}(t)=\prod_{p=0}^{+\infty}\left(\sum_{m=0}^{+\infty} \frac{\left(\mathrm{i} t a_{p}\right)^{m}}{m !} \frac{\mathrm{d}^{p m}}{\mathrm{~d} z^{p m}}\right)=\prod_{p=0}^{\infty} U_{p}\left(a_{p} t\right) .
$$

This operator can actually be regarded as the operator associated with the multiplier given by the function

$$
\hat{U}_{\infty}(\zeta, t):=\prod_{p=0}^{\infty}\left(\sum_{m=0}^{\infty} \frac{\left(\mathrm{i} t a_{p}\right)^{m}}{m !} \zeta^{p m}\right),
$$

which can be rewritten as

$$
\begin{aligned}
\hat{U}_{\infty}(\zeta, t) & =\prod_{p=0}^{\infty}\left(\sum_{m=0}^{\infty} \frac{1}{m !}\left(\mathrm{i} t a_{p} \zeta^{p}\right)^{m}\right) \\
& =\prod_{p=0}^{\infty} \exp \left(\mathrm{i} t a_{p} \zeta^{p}\right) \\
& =\exp \left(\mathrm{i} t \sum_{p=0}^{\infty} a_{p} \zeta^{p}\right) .
\end{aligned}
$$

It is immediate to note that under suitable conditions on the sequence $\left\{a_{p}\right\}$, the function $\hat{U}_{\infty}(\zeta, t)$ is holomorphic in the open disc $|\zeta|<1$ as a function of $\zeta$. This, for example, is true if $a_{p}=1$, for all $p$, in which case $\hat{U}_{\infty}(\zeta, t)=\exp \left(\frac{\mathrm{i} t}{1-\zeta}\right)$. As a consequence, and in view of proposition 3.7 , the operator $U_{\infty}(t)$ acts continuously on the $\operatorname{space}_{\operatorname{Exp}}(\mathbb{C})$. Moreover, every function of the form

$$
Y_{n}(z, a):=\sum_{j=0}^{n} C_{j}(n, a) \mathrm{e}^{\mathrm{i} k_{j}(n) z}
$$

with $\left|k_{j}(n)\right| \leqslant 1$ belong to $\operatorname{Exp}_{1}(\mathbb{C})$.

It is worthwhile to note that under stronger conditions on the sequence $a_{p}$, one can show that the function $\hat{U}_{\infty}(\zeta, t)$ can be rendered on a disc of arbitrary radius $R$. For example, if the sequence $\left\{a_{p}\right\}$ defines an infinite-order differential operator, the function $\sum_{n=0}^{\infty} a_{p} \zeta^{p}$ is entire and so is $\hat{U}_{\infty}(\zeta, t)$.

The preceding discussion leads to the following result.

Theorem 5.1. Let $\left\{a_{p}\right\}$ be a sequence of complex numbers such that the function

$$
\sum_{p=0}^{\infty} a_{p} \zeta^{p}
$$


is analytic in the disc $|\zeta|<R$ for some $R>0$. Then the function

$$
\hat{U}_{\infty}(\zeta, t)=\exp \left(\mathrm{i} t \sum_{p=0}^{\infty} a_{p} \zeta^{p}\right)
$$

is a continuous multiplier on the space of functions analytic in the disc $|\zeta|<R$ and the associated operator

$$
U_{\infty}(t)=\prod_{p=0}^{\infty}\left(\sum_{m=0}^{\infty} \frac{\left(\mathrm{i} t a_{p}\right)^{m}}{m !} \frac{\mathrm{d}^{p m}}{\mathrm{~d} z^{p m}}\right)=\prod_{p=0}^{\infty} U_{p}\left(a_{p} t\right)
$$

acts continuously on the space of entire functions of exponential type less than $R$.

Proof. This is an immediate consequence of proposition 3.8 and the discussion in the previous section.

We can now state our main result, originally proved in [6]:

Theorem 5.2. Let $a \in \mathbb{R}, a>1$. Consider a sequence of complex numbers $\left\{a_{p}\right\}$ such that:

(i) the function $\sum_{p=0}^{\infty} a_{p} \zeta^{p}$ is holomorphic in $\Delta_{a^{\prime}}$ for $a^{\prime}>a$,

(ii) $G(\mathrm{i} a)$ is real and $|G(\mathrm{i} a)| \geqslant a$.

Consider, in the space of entire functions of exponential type less than $a^{\prime}$, the Cauchy problem for the generalized Schrödinger equation

$$
\mathrm{i} \frac{\partial \psi(z, t)}{\partial t}=-G\left(\frac{\mathrm{d}}{\mathrm{d} z}\right) \psi(z, t), \quad \psi(z, 0)=F_{n}(z),
$$

where

$$
G\left(\frac{\mathrm{d}}{\mathrm{d} z}\right)=\sum_{p=0}^{\infty} a_{p} \frac{\mathrm{d}^{p}}{\mathrm{~d} z^{p}}
$$

Then the solution $\psi_{n}(z, t)$, is given by

$$
\psi_{n}(z, t)=\sum_{k=0}^{n} C_{k}(n, a) \mathrm{e}^{-\mathrm{i} z(1-2 k / n)} \mathrm{e}^{\mathrm{i} t G(-\mathrm{i}(1-2 k / n))} .
$$

Moreover, for all fixed t we have

$$
\lim _{n \rightarrow \infty} \psi_{n}(z, t)=\mathrm{e}^{\mathrm{i} t G(\mathrm{i} a)} \mathrm{e}^{\mathrm{i} a z},
$$

and for $z$ on the compact sets of $\mathbb{C}$.

Proof. Here we just sketch the proof. More details are given in [6].

Some computations and the previous method based on the Fourier transform yield

$$
\psi_{n}(z, t)=\sum_{k=0}^{n} C_{k}(n, a) \mathrm{e}^{-\mathrm{i} z(1-2 k / n)} \mathrm{e}^{\mathrm{i} t G(-\mathrm{i}(1-2 k / n))} .
$$

We have

$$
\psi_{n}(z, t)=\sum_{k=0}^{n} C_{k}(n, a) \mathrm{e}^{-\mathrm{i} z(1-2 k / n)} \mathrm{e}^{\mathrm{i} t \sum_{p=0}^{\infty} a_{p}(-\mathrm{i}(1-2 k / n))^{p}}
$$


which can be written as

$$
\begin{aligned}
\psi_{n}(z, t) & =\sum_{k=0}^{n} C_{k}(n, a) \mathrm{e}^{-\mathrm{i} z(1-2 k / n)} \prod_{p=0}^{\infty} \mathrm{e}^{\mathrm{i} t a_{p}(-\mathrm{i}(1-2 k / n))^{p}} \\
& =\sum_{k=0}^{n} C_{k}(n, a) \mathrm{e}^{-\mathrm{i} z(1-2 k / n)} \prod_{p=0}^{\infty} \sum_{m=0}^{\infty} \frac{\left(\mathrm{i} t a_{p}\right)^{m}}{m !}(-\mathrm{i}(1-2 k / n))^{m p} \\
& =\prod_{p=0}^{\infty} \sum_{m=0}^{\infty} \frac{\left(\mathrm{i} t a_{p}\right)^{m}}{m !} \sum_{k=0}^{n} C_{k}(n, a)(\mathrm{i}(1-2 k / n))^{m p} \mathrm{e}^{-\mathrm{i} z(1-2 k / n)}
\end{aligned}
$$

and so we finally have

$$
\begin{aligned}
\psi_{n}(z, t) & =\prod_{p=0}^{\infty} \sum_{m=0}^{\infty} \frac{\left(\mathrm{i} t a_{p}\right)^{m}}{m !} \sum_{k=0}^{n} C_{k}(n, a) \frac{d^{m p}}{d^{m p}} \mathrm{e}^{-\mathrm{i} z(1-2 k / n)} \\
& =\prod_{p=0}^{\infty} \sum_{m=0}^{\infty} \frac{\left(\mathrm{i} t a_{p}\right)^{m}}{m !} \frac{d^{m p}}{d^{m p}} \sum_{k=0}^{n} C_{k}(n, a) \mathrm{e}^{-\mathrm{i} z(1-2 k / n)} \\
& =\prod_{p=0}^{\infty} \sum_{m=0}^{\infty} \frac{\left(\mathrm{i} t a_{p}\right)^{m}}{m !} \frac{d^{m p}}{d^{m p}} F_{n}(z) .
\end{aligned}
$$

Under suitable conditions (see theorem 5.1), we can pass to the limit for $n \rightarrow \infty$ thus obtaining

$\psi(z, t)=\prod_{p=0}^{\infty} \sum_{m=0}^{\infty} \frac{\left(\mathrm{i} t a_{p}\right)^{m}}{m !} \frac{d^{m p}}{d^{m p}} \mathrm{e}^{\mathrm{i} a z}=\prod_{p=0}^{\infty} \sum_{m=0}^{\infty} \frac{\left(\mathrm{i} t a_{p}(\mathrm{i} a)^{p}\right)^{m}}{m !} \mathrm{e}^{\mathrm{i} a z}=\mathrm{e}^{\mathrm{i} t \sum_{p=0}^{\infty}\left(a_{p}(\mathrm{i} a)^{p}\right)} \mathrm{e}^{\mathrm{i} a z}$,

and we have

$$
\lim _{n \rightarrow \infty} \psi_{n}(z, t)=\mathrm{e}^{\mathrm{i} t G(\mathrm{i} a)} \mathrm{e}^{\mathrm{i} a z} .
$$

To conclude, we note that if we set $g(a)=G(\mathrm{i} a)$, and we suitably choose the coefficients $a_{p}$ of the series expressing $G$, we obtain a very large class of superoscillating functions.

\section{A concluding remark}

We point out that recently in the paper [17], the techniques described above have been applied to study the evolution of a superoscillating initial data for the quantum harmonic oscillator. The main result is the following:

Theorem 6.1. Let $F_{n}(x)$ be the superoscillating sequence defined in (1). Then the solution of the Cauchy problem

$$
\mathrm{i} \frac{\partial \psi(x, t)}{\partial t}=\frac{1}{2}\left(-\frac{\partial^{2}}{\partial x^{2}}+x^{2}\right) \psi(t, x), \quad \psi(0, x)=F_{n}(x, a)
$$

is given by

$\psi_{n}(x, t)=(\cos t)^{-1 / 2} \mathrm{e}^{-(\mathrm{i} / 2) x^{2} \tan t} \sum_{k=0}^{n} C_{k}(n, a) \mathrm{e}^{\mathrm{i} x(1-2 k / n) / \cos t-(\mathrm{i} / 2)(1-2 k / n)^{2} \tan t}$.

Moreover, if we set $\psi(t, x)=\lim _{n \rightarrow \infty} \psi_{n}(x, t)$, then we have

$$
\psi(x, t)=(\cos t)^{-1 / 2} \mathrm{e}^{-(\mathrm{i} / 2)\left(x^{2}+a^{2}\right) \tan t+\mathrm{i} a x / \cos t} .
$$

This result shows that these techniques may work also for differential equations with non-constant coefficients. 


\section{Acknowledgments}

The authors are grateful to Professor MV Berry for his useful comments on an earlier version of the paper.

\section{References}

[1] Aharonov Y, Albert D and Vaidman L 1988 How the result of a measurement of a component of the spin of a spin-1/2 particle can turn out to be 100 Phys. Rev. Lett. $601351-4$

[2] Aharonov Y, Colombo F, Nussinov S, Sabadini I, Struppa D C and Tollaksen J 2012 Superoscillation phenomena in SO(3) Proc. R. Soc. Lond. A $4683587-600$

[3] Aharonov Y, Colombo F, Sabadini I, Struppa D C and Tollaksen J 2011 Some mathematical properties of superoscillations J. Phys. A: Math. Theor. 44365304

[4] Aharonov Y, Colombo F, Sabadini I, Struppa D C and Tollaksen J 2013 On some operators associated to superoscillations Complex Anal. Operator Theory 7 1299-310

[5] Aharonov Y, Colombo F, Sabadini I, Struppa D C and Tollaksen J 2013 On the Cauchy problem for the Schrödinger equation with superoscillatory initial data J. Math. Pures Appl. 99 165-73

[6] Aharonov Y, Colombo F, Sabadini I, Struppa D C and Tollaksen J 2013 Superoscillating sequences as solutions of generalized Schrödinger equations (in preparation)

[7] Aharonov Y, Colombo F, Sabadini I, Struppa D C and Tollaksen J 2013 On superoscillations longevity: a windowed Fourier transform approach Quantum Theory: a Two-Time Success Story ed D C Struppa and J Tollaksen (Milan: Springer) pp 313-25

[8] Aharonov Y, Colombo F, Sabadini I, Struppa D C and Tollaksen J The mathematics of superoscillations (in preparation)

[9] Aharonov Y and Rohrlich D 2005 Quantum Paradoxes: Quantum Theory for the Perplexed (Weinheim: Wiley)

[10] Aharonov Y and Vaidman L 1990 Properties of a quantum system during the time interval between two measurements Phys. Rev. A 41 11-20

[11] Balser W 2004 Summability of formal power-series solutions of partial differential equations with constant coefficients J. Math. Sci. 124 5085-97

[12] Berenstein C A and Struppa D C 1988 Dirichlet series and convolution equations Publ. Res. Inst. Math. Sci. Kyoto Univ. 24 783-810

[13] Berry M V 1994 Faster than Fourier Quantum Coherence and Reality: in Celebration of the 60th Birthday of Yakir Aharonov ed J S Anandan and J L Safko (Singapore: World Scientific) pp 55-65

[14] Berry M V 2013 Exact nonparaxial transmission of subwavelength detail using superoscillations J. Phys. A: Math. Theor. 46205203

[15] Berry M and Dennis M R 2009 Natural superoscillations in monochromatic waves in D dimension J. Phys. A: Math. Theor. 42022003

[16] Berry M V and Popescu S 2006 Evolution of quantum superoscillations, and optical superresolution without evanescent waves J. Phys. A: Math. Gen. 39 6965-77

[17] Buniy R, Colombo F, Sabadini I and Struppa D C 2013 Quantum harmonic oscillator with superoscillating initial datum (in preparation)

[18] Ehrenpreis L 1970 Fourier Analysis in Several Complex Variables (New York: Wiley Interscience)

[19] Ichinobe K 2003 Integral representation for Borel sum of divergent solution to a certain nonKowalevski type equation Publ. Res. Inst. Math. Sci. Kyoto Univ. 39 657-93

[20] Kaneko A 1988 Introduction to Hyperfunctions (Dordrecht: Kluwer)

[21] Kowalsevski S 1875 Zur Theorie der partiellen Differentialeichungen J. Reine Angew. Math. 80 1-32

[22] Levin B J 1964 Distribution of Zeros of Entire Functions (Translation of Mathematical Monograph) (Providence, RI: American Mathematical Society)

[23] Lutz D A, Miyake M and Schäfke R 1999 On the Borel summability of divergent solutions of the heat equation Nagoya Math. J. 154 1-29

[24] Taylor B A 1968 Some locally convex spaces of entire functions Entire Functions and Related Parts of Analysis; Proc. Symp. Pure Math. (La Jolla, California, 1966) (Providence, RI: American Mathematical Society) pp 431-67 